\title{
PREVALÊNCIA DE COLONIZAÇÃO POR ESTREPTOCOCO DO GRUPO B E CONHECIMENTO DAS GESTANTES EM RELAÇÃO À IMPORTÂNCIA DA REALIZAÇÃO E PREPARAÇÃO PARA O EXAME
}

\author{
Rafaela Santi DELL'OSBEL, Raiane Tainara da SILVA, Caroline PEZZI, Ramison dos SANTOS, Maria \\ Luisa de Oliveira GREGOLETTO \& Cleber CREMONESE*
}

Centro Universitário da Serra Gaúcha. Caxias do Sul, Rio Grande do Sul, Brasil.

*Autor para correspondência: cleber.cremonese@fsg.br

DOI: http://dx.doi.org/10.18571/acbm.176

\section{RESUMO}

O presente estudo teve como objetivo investigar a prevalência de colonização materna para Streptococcus agalactiae e o conhecimento destas gestantes sobre o exame Estreptococo do Grupo $B$ (EGB), associado a importância da realização, preparação para o exame e consequências para mãe e bebê. Foi realizado um estudo epidemiológico transversal com uma amostra de 215 gestantes, usuárias do SUS na cidade de Caxias do Sul, entre fevereiro e julho de 2016. Amostras biológicas para a detecção de colônias foram realizadas em laboratório credenciado pelo SUS, com aplicação de questionário para medir o nível de conhecimento. Análise descritiva, prevalência e razão de prevalência bruta e ajustada foram aplicadas para caracterizar a amostra e identificar possíveis diferenças entre variáveis de exposição, com aplicação de teste Qui-quadrado para heterogeneidade e Teste $\mathrm{t}$ de Student, com nível de significância $\mathrm{p} \leq 0,05$. A prevalência de colonização materna para Streptococcus agalactiae foi de 19,5\%, porém sem associações significativas. Prevalência geral para conhecimento satisfatório sobre aspectos do exame foi de $78,6 \%$, onde $\geq 9$ anos de escolaridade ( $\mathrm{RP}=1,18$; IC95\% 1,02-1,36; $\mathrm{p}=0,022$ ) e residir com $\geq 4$ pessoas na casa $(\mathrm{RP}=1,20$; IC95\% 1,05-1,38; $\mathrm{p}=0,007)$ apresentaram-se como fatores associados ao desfecho. A prevalência geral para exames positivos foi semelhante a outros estudos, porém não foi possível identificar fatores de risco. Ainda, identificou-se um elevado conhecimento das gestantes sobre o exame e sua importância. Diante dos resultados, sugere-se continuação e implementação de ações que possibilitem suporte adequado para gestantes e bebês.

Palavras-chave: Gestantes; Streptococcus agalactiae; Conhecimento; Cuidado Pré-Natal; Estudos Transversais.

\section{ABSTRACT}

Colonization prevention by Group B Estrephococcus and knowledge of pregnant women regarding the importance of the realization and preparation for the examination. The present study aimed to investigate the prevalence of maternal colonization for Streptococcus agalactiae and the knowledge of these pregnant women about the Group B Streptococcus (GBS), associated with the importance of the achievement, preparation for the examination and consequences for mother and baby. A Cross-sectional epidemiological study was carried out with a sample of 215 pregnant women, SUS users in the city of Caxias do Sul, between February and July 2016. Biological samples for the detection of colonies were performed in a laboratory accredited by the SUS, with a questionnaire for measure the level of knowledge. Descriptive analysis, prevalence and crude and adjusted prevalence ratio were applied to characterize the sample and to identify possible differences between exposure variables, with application of Chi-square test for heterogeneity and Student's t-test, with significance level of $\mathrm{p} \leq 0.05$. The prevalence of maternal colonization for Streptococcus agalactiae was $19.5 \%$, but without significant associations. General prevalence for satisfactory knowledge on aspects of the exam was $78.6 \%$, where $\geq 9$ years 


\section{Biomedica Brasiliensia}

ISSN: 2236-0867

of schooling ( $\mathrm{PR}=1.18 ; 95 \% \mathrm{CI} 1.02-1.36 ; \mathrm{p}=0.022)$ and resided with $\geq 4$ people in the household $(\mathrm{PR}=1.20 ; 95 \% \mathrm{CI} 1.05-1.38 ; \mathrm{p}=0.007)$ presented as factors associated with the outcome. The overall prevalence for positive tests was similar to other studies, but it was not possible to identify risk factors. Also, a high knowledge of pregnant women about the examination and its importance was identified. In view of the results, it is suggested to continue and implement actions that allow adequate support for pregnant women and babies.

Keywords: Pregnant women; Streptococcus agalactiae; Knowledge; Prenatal Care; CrossSectional Studies.

\section{Introdução}

Estreptococo $\beta$ - hemolítico ou grupo B (EGB) é um anaeróbio Gram-positivo em forma de cocos, o qual integra a microbiota humana, colonizando geralmente o trato gastrintestinal e geniturinário (DARABI et al., 2017). Em mulheres, no período gestacional, essa colonização é considerada como um fator de risco para infecções subsequentes ao neonato, uma vez que por meio da transmissão vertical do micro-organismo pelo canal vaginal podem ocorrer quadros de septicemia, meningite e pneumonia (DARABI et al., 2017; VACILOTO et al., 2002). No caso da cultura para EGB positivar, é realizada a administração profilática de antibióticos a fim de evitar a contaminação, bem como suas possíveis complicações no bebê (JI et al., 2017; VORNHAGEN, WALDORF e RAJAGOPAL, 2017).

A prevalência de colonização materna por EGB pode estar associada a raça, idade materna, paridade, alto índice de massa corporal e nível socioeconômico (DARABI et al., 2017). Ainda, a precisão da detecção da colonização depende da densidade, da escolha do método bacteriológico e do número de culturas obtidas (VACILOTO et al., 2002; VORNHAGEN, WALDORF e RAJAGOPAL, 2017). Devido a tais fatores, a taxa de colonização por EGB varia para diferentes regiões do mundo, como na Índia, Irã, Suíça e Marrocos, onde a prevalência é respectivamente 7,6\%, 11,8\%, 21\% e 24\% (DARABI et al., 2017; MORALEDA et al., 2018; RAUSCH et al., 2017; SANTHANAM et al., 2017). No Brasil a prevalência geral varia de 10 a 30\%, com variação entre as diferentes regiões do país (JI et al., 2017; LINHARES et al., 2011; PENELAS e PINHEIRO, 2016). Relacionado ao controle da infecção neonatal por EGB, é mencionado, nas recomendações do Centers for Disease Control and Prevention (CDC), a realização da triagem universal nas $35^{\text {a }}$ e $37^{a}$ semanas de gestação e a quimioprofilaxia intraparto, baseada em fatores de risco gestacionais (CONTROL e PREVENTION, 2011). Porém, observa-se que não há a prática regular destas indicações (FUNÇÃO e NARCHI, 2013).

O fortalecimento nas orientações para as gestantes deve se dar por meio da incorporação destas condutas acolhedoras, além do desenvolvimento de ações educativas e preventivas aos exames pré-natais, como por exemplo, uma melhoria na abordagem referente à infecção por EGB (PAVANATTO e ALVES, 2014). Nestas circunstâncias, a atenção humanizada à mulher gestante deve estender-se desde o pré-natal, passando ao parto até o puerpério. Esta atenção segue sendo um dos grandes desafios para os países em desenvolvimento dentro do contexto social, educacional e de acesso (ELBARADIE, MAHMOUD e FARID, 2009; KRUK et al., 2013; SHARMILA et al., 2011). Embora haja o fortalecimento da atenção para gestantes quanto à clínica que antecede o parto, ainda se evidencia a inadequação na falta de orientações e assistência no que diz respeito aos principais exames laboratoriais realizados no pré-natal, incluindo a pesquisa por EGB (BRASIL, 2000). Além disso, sabe-se que o nível de conhecimento sobre EGB entre as gestantes é menor comparado a outras infecções relativas ao mesmo período. Considera-se a importância do conhecimento sobre o exame para a realização do mesmo, observando-se a necessidade de abordar a importância do conhecimento sobre EGB, visando garantir a realização do exame e prevenir complicações associadas (PAVANATTO e ALVES, 2014; YOUDEN et al., 2005). 
Considerando a relevância do tema envolvendo a compreensão sobre o EGB no pré- natal e fatores relacionados às inadequações deste acesso, o presente estudo teve como objetivo investigar a prevalência de colonização materna para Streptococcus agalactiae e o conhecimento destas gestantes sobre o exame Estreptococo do Grupo B (EGB), associado a importância da realização, preparação para o exame e consequências para mãe e bebê.

\section{Materiais e Métodos}

\subsection{Delineamento e população de estudo}

Trata-se de um estudo epidemiológico analítico observacional, com delineamento transversal, composto por 215 gestantes que realizaram acompanhamento pré-natal em 17 unidades básicas de saúde (UBS’s) na cidade de Caxias do Sul - RS.

\subsection{Característica amostral}

A amostra foi formada por conveniência, entre fevereiro e julho de 2016, sendo convidadas a participar da pesquisa todas as gestantes com idade $\geq 18$ anos, entre a $30^{\mathrm{a}}$ e $40^{\mathrm{a}}$ semanas de gestação, encaminhadas a um laboratório central do município de Caxias do Sul, conveniado ao SUS, as quais tinham por objetivo realizar o exame EGB para triagem da presença por colonização de Streptococcus agalactiae. Quanto aos critérios de inclusão, era necessário que a gestante apresentasse condições físicas para responder a um questionário autoaplicável, o qual continha questões referentes aos objetivos da pesquisa. Gestantes analfabetas, com idioma de origem diferente do português, com idade inferior a 18 anos ou que apresentassem total dificuldade na leitura e interpretação das questões, de forma que as impossibilitasse de responder ao questionário, foram excluídas do processo. Da mesma forma, não participaram da pesquisa as gestantes que não realizaram o preparo solicitado pelo laboratório para a realização do EGB, sendo este um critério de segurança para evitar resultados falso-negativos.

\subsection{Coleta de dados - Material biológico}

As coletas de material vaginal e anal foram realizadas por meio de swab estéril, sem colocação de espéculo, evitando contaminação com áreas adjacentes, por uma técnica responsável do laboratório. Imediatamente após a coleta, o swab foi inoculado em um tubo de vidro contendo caldo granada bifásico ${ }^{\mathrm{TM}}$, um meio seletivo utilizado para a detecção e a identificação direta de Streptococcus agalactiae. Posteriormente, o tubo foi incubado por 24 horas em estufa a $37^{\circ} \mathrm{C}$. Quando o EGB não foi identificado após 24 horas de incubação, o material foi incubado novamente por mais 24 horas, e a leitura final efetuada após 48 horas. Por fim, o resultado foi interpretado a partir da mudança de cor do meio de cultura, sendo positivo quando apresentasse a cor laranja/vermelho.

\subsection{Coleta de dados - Questionário}

Para a coleta de dados, utilizou-se um questionário autoaplicável, construído pelos pesquisadores responsáveis e condizente com o planejamento e execução do trabalho. A confiabilidade do instrumento utilizado para coleta de dados foi realizada por meio de um teste piloto, realizado no mesmo laboratório, no mês de janeiro de 2016, no qual foram entrevistadas 5 gestantes (não inclusas na amostra final). O instrumento de pesquisa consistia de questões sociodemográficas, de acesso à informação, características gestacionais, bem como variáveis relacionadas ao nível de conhecimento das gestantes sobre a importância, preparação e consequências da realização do exame de pesquisa de EGB. 
Para as informações sociodemográficas as variáveis estudadas foram: idade (coletada de forma contínua e categorizada através do valor da média em $\leq 24$ anos e $\geq 25$ anos), escolaridade (coletada de forma contínua e categorizada através do valor da média em $\leq 8$ anos de estudo e $\geq 9$ anos de estudo), cor de pele (autodeclarada e categorizada em não branca e branca), estado civil (categorizada em solteira/outros e casada/união estável) e renda (categorizada em: sem renda até 1 salário mínimo e > 1 salário mínimo). As características de moradia coletadas foram: pessoas na casa (coletada de forma contínua e categorizada através do valor da média em $\leq 3$ pessoas e $\geq 4$ pessoas) e área que reside (rural ou urbana). Ainda, a profissão das gestantes foi coletada de forma aberta (e posteriormente categorizada em do lar/desempregada e trabalhadora formal/autônoma). Em relação às variáveis gestacionais, foram coletadas: semana gestacional (coletada de forma contínua e categorizada através do valor da média em $\leq 35$ semanas e $\geq 36$ semanas), número de gestações (uma e duas ou mais), diagnóstico de DST's (sim e não), presença de doença crônica ( média em $\leq 7$ e $\geq 8$ ) (Tabela 1 ).

Para obter informações relacionadas ao desfecho "Conhecimento das gestantes sobre o exame Estreptococo do Grupo B (EGB), associado a importância da realização, preparação para o exame e consequências para mãe e bebê" foram inseridas no questionário 10 questões afirmativas, sendo elas: 1) O exame tem objetivo de detectar a bactéria Estreptococo do grupo B na região da vagina da gestante; 2) $\mathrm{O}$ risco de infecção por Estreptococo do grupo B ocorre somente nas gestantes, sem riscos ao bebê; 3) O exame identifica inúmeras Doenças Sexualmente Transmissíveis (DST); 4) O tratamento, no caso de resultado positivo para Estreptococos do grupo $\mathrm{B}$, é realizado somente no pós-parto; 5) O exame positivo para Estreptococos do grupo B em gestantes, sem tratamento, tem associação com meningite no recém- nascido; 6) O exame para Estreptococos do grupo B deveria ser realizado também nos homens (parceiros das gestantes); 7) O exame para Estreptococos do grupo B poderá identificar problemas de saúde no bebê antes do parto; 8) Caso a gestante apresente exame positivo, a contaminação ao bebê pode ocorrer no momento do parto; 9) A gestante poderá fazer higiene íntima e urinar antes da coleta, sem nenhum problema no resultado do exame; 10) Se o exame para estreptococo for positivo é recomendado o uso de pomadas vaginais ou cremes vaginais. As gestantes podiam responder a estas afirmações, assinalando apenas "sim" ou não". Todas as 10 questões continham apenas uma possibilidade de resposta correta (Quadro 1), com um ponto por acerto.

Assim, cada gestante apresentou uma pontuação total. A classificação do desfecho foi realizada através da somatória do número de acertos, para as 10 frases afirmativas, onde aquelas gestantes que apresentaram o número de $\geq 6$ pontos/acertos (valor da média geral) foram classificadas como conhecimento satisfatório. 


\begin{tabular}{|c|c|}
\hline Frases afirmativas & Resposta correta \\
\hline $\begin{array}{l}\text { O exame tem objetivo de detectar a bactéria Estreptococo do grupo B na região da vagina da } \\
\text { gestante. }\end{array}$ & Sim \\
\hline $\begin{array}{l}\text { O risco de infecção por Estreptococo do grupo B ocorre somente nas gestantes, sem riscos ao } \\
\text { bebê. }\end{array}$ & Não \\
\hline O exame identifica inúmeras doenças sexualmente transmissíveis (DST). & Não \\
\hline $\begin{array}{l}\text { O tratamento, no caso de resultado positivo para Estreptococos do grupo B, é realizado } \\
\text { somente no pós-parto. }\end{array}$ & Não \\
\hline $\begin{array}{l}\text { O exame positivo para Estreptococos do grupo B em gestantes, sem tratamento, tem } \\
\text { associação com meningite no recém-nascido. }\end{array}$ & Sim \\
\hline $\begin{array}{l}\text { O exame para Estreptococos do grupo B deveria ser realizado também nos homens } \\
\text { (parceiros das gestantes). }\end{array}$ & Não \\
\hline $\begin{array}{l}\text { O exame para Estreptococos do grupo B poderá identificar problemas de saúde no bebê antes } \\
\text { do parto. }\end{array}$ & Não \\
\hline $\begin{array}{l}\text { Caso a gestante apresente exame positivo, a contaminação ao bebê pode ocorrer no momento } \\
\text { do parto. }\end{array}$ & Sim \\
\hline $\begin{array}{l}\text { A gestante poderá fazer higiene íntima e urinar antes da coleta sem nenhum problema no } \\
\text { resultado do exame. }\end{array}$ & Não \\
\hline $\begin{array}{l}\text { Se o exame para Estreptococo for positivo é recomendado o uso de pomadas vaginais ou } \\
\text { cremes vaginais. }\end{array}$ & Não \\
\hline
\end{tabular}

Quadro 1: Afirmativas utilizadas para medir o desfecho "Conhecimento do exame EGB (importância da realização, preparação para o exame e consequências para mãe e bebê), entre mulheres gestantes, atendidas em UBS's”. Caxias do Sul, 2016.

\subsection{Análises de dados}

A análise descritiva das variáveis estudadas foi realizada por meio de valores absolutos, frequências e prevalências do desfecho. A análise estatística foi efetuada por meio do programa SPSS Statistic Data 23, onde as médias para conhecimento satisfatório e insatisfatório foram realizadas com suporte do Teste-t de Student para amostras independentes. Já as análises bivariadas foram realizadas por meio do teste de Qui-quadrado, para comparação de variáveis categóricas. A análise bruta foi realizada através do STATA 11 e apresentou as razões de prevalência e respectivos IC 95\%. Ainda, na análise multivariada ou ajustada realizou-se regressão de Poisson, como resultado encontra-se a razão de prevalência. Para a análise ajustada utilizou-se a técnica de backwards, seguindo o critério: as variáveis que atingiram até $20 \%$ de significância $(\mathrm{p} \leq 0,20)$ na análise bruta foram inseridas no modelo final (Tabela 3$)$, onde considerou-se um nível de significância de $5 \%(\mathrm{p} \leq 0,05)$ para identificar associação entre o desfecho e as variantes de exposição.

\subsection{Aspectos éticos}

O projeto de pesquisa foi aprovado pelo Comitê de Ética em Pesquisa do Centro Universitário da Serra Gaúcha, de acordo com a Resolução nº66/12 do Conselho Nacional de Saúde, sob parecer $\mathrm{n}^{\mathrm{o}}$ 1.360.002. Todas as participantes do estudo leram e assinaram o Termo de 
Consentimento Livre e Esclarecido (TCLE), o qual autorizou a participação e publicação dos resultados em anonimato.

\section{Resultados}

O estudo foi composto por 215 gestantes, usuárias do SUS, da cidade de Caxias do SulRS. A idade média das gestantes foi de 24,7( $\pm 5,6)$ anos, com 59,1\% apresentando até 24 anos. Gestantes com até 8 anos de estudo, brancas e casadas ou em união estável, representaram, respectivamente, $49,8 \%, 51,6 \%$ e $58,1 \%$ das entrevistadas. Apresentaram renda familiar maior ou igual a um salário mínimo ( $\mathrm{R} \$ 880,00), 73,1 \%$ das mulheres. Referindo-se a área que residiam, constatou-se que $93,1 \%$ vivam no perímetro urbano da cidade, e 55,9\% das gestantes se disseram do lar ou desempregadas. Quando se observou as características gestacionais, aponta-se que as mulheres com duas ou mais gestações e $\leq 35$ semanas, representaram 56,3\% e 53,5\% respectivamente. A média de consultas pré-natal foi de 7,73( $\pm 5,6)$.

A prevalência de colonização materna para Streptococcus agalactiae foi de 19,5\% na população estudada. Verificou-se que mulheres com idade entre 18 e 24 anos, solteiras, com renda de até um salário mínimo, que residiam na área rural e que estavam na primeira gestação, apresentaram maiores prevalências de resultados positivos, porém não houve diferença estatisticamente significativa em relação à prevalência de exames positivos para EGB e as variáveis de exposição $(\mathrm{p}>0,05)$ (Tabela 1). 
Tabela 1: Descrição da amostra e prevalência de exames positivos para EGB ${ }^{\dagger}$, entre mulheres gestantes atendidas em Unidades Básicas de Saúde. Caxias do Sul. 2016. (n=215).

\begin{tabular}{|c|c|c|c|}
\hline Variáveis de exposição & n $(\%)$ & $\begin{array}{c}\text { Prevalência de exames } \\
\text { positivos }(\%)\end{array}$ & p-valor* \\
\hline Idade $(X=24,73 \pm 5,6)$ & & & 0,947 \\
\hline$\leq 24$ anos & $127(59,1)$ & 19,7 & \\
\hline$\geq 25$ anos & $88(40,9)$ & 19,3 & \\
\hline Escolaridade & & & 0,973 \\
\hline$\leq 8$ anos & $107(49,8)$ & 19,6 & \\
\hline$\geq 9$ anos & $108(50,2)$ & 19,4 & \\
\hline Cor de pele & & & 0,650 \\
\hline Não branca & $104(48,4)$ & 18,3 & \\
\hline Branca & $111(51,6)$ & 20,7 & \\
\hline Estado civil & & & 0,884 \\
\hline Solteira e outros & $90(41,9)$ & 20,0 & \\
\hline Casada ou união estável & $125(58,1)$ & 19,2 & \\
\hline Renda & & & 0,070 \\
\hline Sem renda até 1 salário mínimo & $58(26,9)$ & 27,6 & \\
\hline > 1 salário mínimo & $157(73,1)$ & 16,6 & \\
\hline Pessoas na casa & & & 0,259 \\
\hline$\leq 3$ & $134(62,3)$ & 17,2 & \\
\hline$\geq 4$ & $81(37,7)$ & 23,5 & \\
\hline Área que reside & & & 0,162 \\
\hline Rural & $15(6,9)$ & 33,3 & \\
\hline Urbana & $200(93,1)$ & 18,5 & \\
\hline Profissão & & & 0,398 \\
\hline Do lar/desempregada & $120(55,9)$ & 17,5 & \\
\hline Trabalhadora formal ou autônoma & $95(44,1)$ & 22,1 & \\
\hline Semana gestacional $(X=35,05 \pm 2,1)$ & & & 0,232 \\
\hline$\leq 35$ semanas & $115(53,5)$ & 16,5 & \\
\hline$\geq 36$ ou mais semanas & $100(46,5)$ & 23,0 & \\
\hline Quantas gestações & & & 0,360 \\
\hline Primeira & $94(43,7)$ & 22,3 & \\
\hline Duas ou mais & $121(56,3)$ & 17,4 & \\
\hline Diagnóstico de DST & & & 0,165 \\
\hline Sim & $23(10,7)$ & 8,7 & \\
\hline Não & $192(89,3)$ & 20,8 & \\
\hline Presença de doença crônica & & & 0,221 \\
\hline $\operatorname{Sim}$ & $6(2,8)$ & 0,0 & \\
\hline Não & $209(97,2)$ & 20,1 & \\
\hline Consultas pré-natal $(X=7,73 \pm 5,6)$ & & & 0,656 \\
\hline$\leq 7$ & $109(50,7)$ & 18,3 & \\
\hline$\geq 8$ & $106(49,3)$ & 20,8 & \\
\hline Exame EGB & & & - \\
\hline Positivo & $42(19,5)$ & - & \\
\hline Negativo & $173(80,5)$ & - & \\
\hline
\end{tabular}

O desfecho de interesse do presente estudo é demonstrado na Figura 1, descrevendo assim, os resultados do conhecimento. Este é representado em 10 afirmativas que foram utilizadas para medir as frequências de respostas satisfatórias/corretas e insatisfatórias/incorretas. A prevalência geral do desfecho investigado, conhecimento satisfatório, foi de 78,6\% (valor não apresentado em tabelas e gráficos). Quando medidas de forma individual, frequências elevadas de conhecimento satisfatório foram encontradas nas questões que abordaram a higiene íntima, como o ato de urinar antes da coleta $(95,3 \%)$, e na questão que abordava o objetivo do exame $(91,6 \%)$. Por outro lado, destaca-se a baixa prevalência de conhecimento em relação às variáveis referentes ao uso de pomadas ou cremes vaginais, caso o exame fosse positivo para EGB $(42,8 \%)$ e principalmente a 
afirmativa que mencionava a realização do exame de EGB em homens, parceiros de gestantes $(37,70 \%)$.

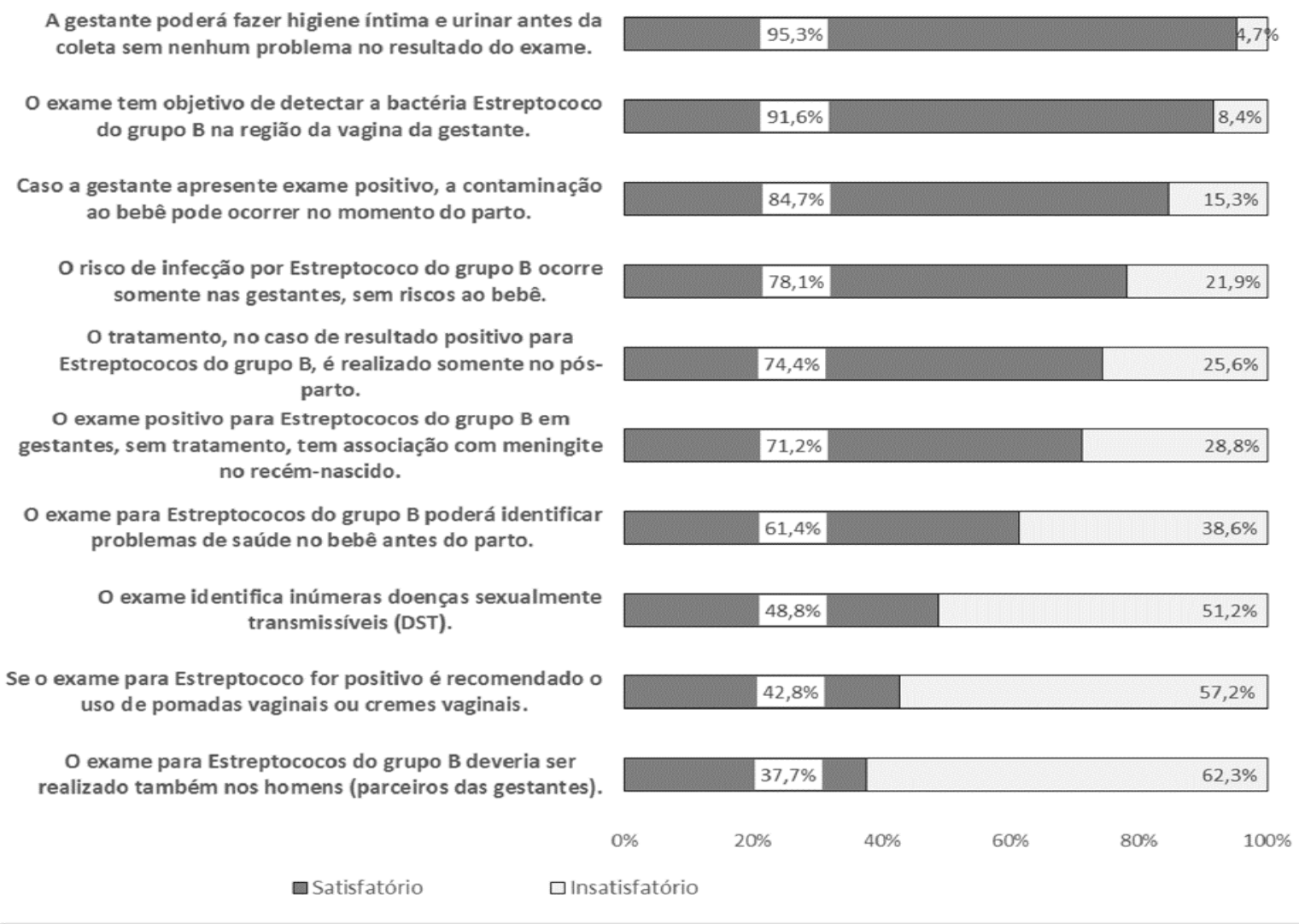

Figura 1: Conhecimento do exame EGC, entre mulheres gestantes, atendidas em UBS's na Cidade de Caxias do Sul-RS. 2016.

Quanto à importância da realização e preparo para o exame, além das consequências para mãe e bebê, ou seja, conhecimento satisfatório do EGB, a Tabela 2 apresentou comportamento semelhante em relação a maiores pontuações médias de acertos e frequências mais elevadas de conhecimento satisfatório, associadas as variáveis de exposição. Quando comparadas as médias de acertos, nota-se diferença significativa entre gestantes com $\geq 9$ anos de estudo $(\mathrm{p}=0,001)$ e autodeclaradas brancas $(\mathrm{p}=0,016)$. Com relação a escolaridade, $84,3 \%$ das gestantes com $\geq 9$ anos de estudo apresentaram conhecimento satisfatório, enquanto no grupo de menor escolaridade este valor foi de 72,9\% ( $\mathrm{p}=0,042$ ). Mulheres com renda maior que 1 salário mínimo e que residiam com $\geq 4$ pessoas na casa no momento da entrevista apresentaram maior percentual de conhecimento satisfatório, respectivamente $82,2 \%$ e $86,4 \%$, com p-valor significativo para conhecimento satisfatório. Não foram encontrados resultados significativos nas demais variáveis de exposição. 
Tabela 2: onhecimento sobre exame EGB (importância da realização, preparação para o exame e consequências para mãe e bebê) através de pontuação média e conhecimento satisfatório ${ }^{\dagger}$, entre mulheres gestantes, atendidas em um UBSs. Caxias do Sul. 2016. $(\mathrm{n}=215)$.

\begin{tabular}{|c|c|c|c|c|}
\hline Variáveis de exposição & $\begin{array}{l}\text { Pontuação } \\
\text { média }\end{array}$ & p-valor* & $\begin{array}{c}\text { Conhecimento } \\
\text { Satisfatório (\%) }\end{array}$ & p-valor** \\
\hline Idade & & 0,105 & & 0,195 \\
\hline$\leq 24$ anos & 6,7 & & 75,6 & \\
\hline$\geq 25$ anos & 7,1 & & 83,0 & \\
\hline Escolaridade & & 0,001 & & 0,042 \\
\hline$\leq 8$ anos & 6,5 & & 72,9 & \\
\hline$\geq 9$ anos & 7,2 & & 84,3 & \\
\hline Cor de pele & & 0,016 & & 0,212 \\
\hline Não branca & 6,6 & & 75,0 & \\
\hline Branca & 7,1 & & 82,0 & \\
\hline Estado civil & & 0,235 & & 0,355 \\
\hline Solteira e outros & 6,7 & & 75,6 & \\
\hline Casada ou união estável & 7,0 & & 80,8 & \\
\hline Renda & & 0,063 & & $\mathbf{0 , 0 3 6}$ \\
\hline Sem renda até 1 salário mínimo & 6,5 & & 69,0 & \\
\hline > 1 salário mínimo & 7,0 & & 82,2 & \\
\hline Pessoas na casa & & 0,767 & & $\mathbf{0 , 0 3 0}$ \\
\hline$\leq 3$ & 6,8 & & 73,9 & \\
\hline$\geq 4$ & 6,9 & & 86,4 & \\
\hline Profissão & & 0,417 & & 0,147 \\
\hline Do lar/desempregada & 6,8 & & 75,0 & \\
\hline Trabalho formal/autônoma & 7,0 & & 83,2 & \\
\hline Semana gestacional & & 0,136 & & 0,593 \\
\hline$\leq 35$ semanas & 7,0 & & 80,0 & \\
\hline$\geq 36$ ou mais semanas & 6,7 & & 77,0 & \\
\hline Quantas gestações & & 0,267 & & 0,970 \\
\hline Primeira & 7,0 & & 78,7 & \\
\hline Duas ou mais & 6,8 & & 78,5 & \\
\hline Diagnóstico de DST & & 0,756 & & 0,620 \\
\hline Sim & 7,0 & & 82,6 & \\
\hline Não & 6,9 & & 78,1 & \\
\hline Presença de doença crônica & & 0,122 & & 0,775 \\
\hline $\operatorname{Sim}$ & 7,8 & & 83,3 & \\
\hline Não & 6,8 & & 78,5 & \\
\hline Consultas pré-natal & & 0,287 & & 0,155 \\
\hline$\leq 7$ & 7,0 & & 82,6 & \\
\hline$\geq 8$ & 6,7 & & 75,5 & \\
\hline Exame EGB & & 0,988 & & 0,405 \\
\hline Positivo & 6,9 & & 83,3 & \\
\hline Negativo & 6,9 & & 77,5 & \\
\hline
\end{tabular}

* Teste t-Student para amostras independentes; ** Teste Qui Quadrado para heterogeneidade; Valores em negrito são estatisticamente significativos $(\mathrm{p} \leq 0,05)$. Conhecimento satisfatório $\geq 6$ acertos das 10 afirmações apresentadas no questionário.

Na Tabela 3 são apresentadas as Razões de Prevalência (RP) brutas, com os resultados considerados significativos (idade, escolaridade, renda, pessoas na casa, profissão, consultas prénatal), utilizados para montar o modelo ajustado $(\mathrm{p} \leq 0,20)$. Demais características investigadas não apresentaram valores significativos para o teste qui-quadrado na análise bruta. Na análise ajustada, o modelo final apresentou valores significativos $(\mathrm{p} \leq 0,05)$ nas variáveis: escolaridade e pessoas na casa. Assim, aquelas gestantes com $\geq 9$ anos de estudo apresentaram uma prevalência $18 \%$ maior de conhecimento satisfatório quanto ao exame EGB, em relação as de menor escolaridade. Por fim, mulheres que residiam com $\geq 4$ pessoas obtiveram $20 \%$ a mais de conhecimento satisfatório quando comparadas as que residiam com $\leq 3$ pessoas. Ainda, gestantes com mais idade, maior renda e com 
trabalho formal apresentaram associação com conhecimento sobre o exame EGB, porém essa não foram estatisticamente significativa.

Tabela 3: Razão de prevalência bruta e ajustada sobre o conhecimento do exame EGB (importância da realização, preparação para o exame e consequências para mãe e bebê), entre mulheres gestantes, atendidas em um UBSs. Caxias do Sul. 2016. $(\mathrm{n}=215)$.

\begin{tabular}{|c|c|c|c|c|}
\hline Variáveis & $\begin{array}{l}\text { RP buta } \\
\text { (IC 95\%) }\end{array}$ & p-valor* & $\begin{array}{l}\text { RP ajustada } \\
\text { (IC 95\%) }\end{array}$ & p-valor* \\
\hline Idade & & 0,184 & & 0,661 \\
\hline$\leq 24$ anos & 1 & & 1 & \\
\hline$\geq 25$ anos & $1,09(0,95-1,25)$ & & $1,03(0,89-1,18)$ & \\
\hline Escolaridade & & 0,045 & & $\mathbf{0 , 0 2 2}$ \\
\hline$\leq 8$ anos & 1 & & 1 & \\
\hline$\geq 9$ anos & $1,14(1,00-1,33)$ & & $1,18(1,02-1,36)$ & \\
\hline Cor de pele & & 0,217 & & \\
\hline Não branca & 1 & & & \\
\hline Branca & $1,09(0,94-1,25)$ & & & \\
\hline Estado civil & & 0,366 & & \\
\hline Solteira e outros & 1 & & & \\
\hline Casada ou união estável & $1,06(0,92-1,23)$ & & & \\
\hline Renda & & 0,068 & & 0,122 \\
\hline Sem renda até $1 \mathrm{SM}$ & 1 & & 1 & \\
\hline > 1 salário mínimo & $1,19(0,98-1,43)$ & & $1,16(0,95-1,41)$ & \\
\hline Pessoas na casa & & $\mathbf{0 , 0 2 1}$ & & $\mathbf{0 , 0 0 7}$ \\
\hline$\leq 3$ & 1 & & 1 & \\
\hline$\geq 4$ & $1,16(1,02-1,33)$ & & $1,20(1,05-1,38)$ & \\
\hline Profissão & & 0,142 & & 0,804 \\
\hline Do lar/desempregada & 1 & & 1 & \\
\hline Trabalho formal/autônoma & $1,10(0,96-1,27)$ & & $1,01(0,87-1,18)$ & \\
\hline Semana gestacional & & 0,596 & & \\
\hline$\leq 35$ semanas & 1 & & & \\
\hline$\geq 36$ ou mais semanas & $0,96(0,83-1,10)$ & & & \\
\hline Quantas gestações & & 0,970 & & \\
\hline Primeira & 1 & & & \\
\hline Duas ou mais & $0,99(0,86-1,14)$ & & & \\
\hline Diagnóstico de DST & & 0,589 & & \\
\hline Sim & 1 & & & \\
\hline Não & $0,94(0,77-1,15)$ & & & \\
\hline Presença de doença crônica & & 0,747 & & \\
\hline $\operatorname{Sim}$ & 1 & & & \\
\hline Não & $0,94(0,65-1,35)$ & & & \\
\hline Consultas pré-natal & & 0,155 & & 0,092 \\
\hline$\leq 7$ & 1 & & 1 & \\
\hline$\geq 8$ & $0,90(0,78-1,03)$ & & $0,88(0,77-1,01)$ & \\
\hline Exame EGB & & 0,363 & & \\
\hline Positivo & 1 & & & \\
\hline Negativo & $0,92(0,79-1,08)$ & & & \\
\hline \multicolumn{5}{|c|}{$\begin{array}{l}\text { RP - Razão de Prevalência. Modelo final ajustado pelo método de regressão de Poisson, por todas as variáveis que } \\
\text { apresentaram p-valor } \leq 0,20 \text { na análise bruta. Variáveis inseridas no modelo: idade, escolaridade, renda, pessoas na } \\
\text { casa, profissão e consultas pré-natal; ajustadas pela técnica de backwards. SM - Salário Mínimo }(\mathrm{R} \$ 880,00) \text {. } \\
* \text { Valores em negrito são estatisticamente significativos }(\mathrm{p} \leq 0,05) \text {. }\end{array}$} \\
\hline
\end{tabular}

\section{Discussão}

O estudo mediu a prevalência de colonização materna para EGB, onde encontrou-se uma prevalência geral de 19,5\%. Em âmbito internacional, esta prevalência encontrada assemelha-se a um estudo publicado recentemente, realizado em Portugal, com 6687 gestantes, com prevalência de $21 \%$ para exames positivos de EGB (PINTO et al., 2017). Ainda, corroboram com estes achados 
o resultado de um artigo realizado na Etiópia com 281 gestantes, verificando-se uma prevalência de 14,6\% (ASSEFA et al., 2018). Ao mesmo tempo, em estudo realizado Taiwan com 154.088 mulheres, a prevalência foi de $19.58 \%$ (HUNG et al., 2018), assemelhando-se a encontrada no presente artigo. Já em nível nacional, um estudo recente, com gestantes do Paraná, apresentou prevalência de 28,4\% (MELO et al., 2018). Em âmbito regional, destaca-se um estudo realizado em Caxias do Sul, com 204 gestantes, estando a prevalência próxima aos valores do estudo, os quais variam de 22,5\% (vaginal) a 26\% (perianal) (WOLLHEIM et al., 2017). Da mesma forma, a prevalência aqui encontrada condiz com os valores presentes na literatura, os quais variam entre $10 \%$ a $30 \%$, estando a maioria acima de 20\% (AREAL et al., 2010; BARCAITE et al., 2008; MENGIST et al., 2017; PENELAS e PINHEIRO, 2016; PINTO et al., 2017).

De acordo com estudo realizado na Etiópia com 281 gestantes, o único fator de risco encontrado para a presença de EGB foi a Instituição de Saúde, embora esta não correspondesse a setores privados ou públicos (ASSEFA et al., 2018). Por outro lado, em um estudo realizado no Marrocos com 350 mulheres grávidas, houve associação para a colonização por EGB com hipertensão arterial gestacional e prurido vaginal (MORALEDA et al., 2018). Porém, estes estudos não apresentaram fatores de risco sociodemográficos ou de estilo de vida para a presença de EGB. Ainda, segundo estudo realizado na Cidade da Guatemala com 896 gestantes, a maior idade apresentava elevado risco para presença de EGB, onde a chance aumentava em 5\% para cada ano da gestante, embora este resultado não coincida aos encontrados na literatura. No mesmo estudo, o aumento da paridade demonstrou uma associação inversa com o risco de colonização (RICK et al., 2017). Ressalta-se a escassa quantidade de artigos que encontraram fatores de risco sociodemográficos para a presença de EGB e pressupõem-se que estes variem de acordo com as características da população estudada. Acredita-se que a elevada quantidade de gestações atuem como efeito protetor para a colonização por EGB, porém a implicação disto ainda não está clara, sugere-se o desenvolvimento de novos estudos.

Destaca-se que neste caso não foram encontradas associações significativas com as variáveis de exposição estudadas. Algumas hipóteses sobre as associações com o desfecho são apresentadas em estudos internacionais, como Hall e Sigaúque, os quais sugerem que exames positivos para EGB estejam relacionados ao sistema imunológico materno (HALL et al., 2017; SIGAÚQUE et al., 2018) e possivelmente não apresente associações com variáveis sociodemográficas que consiga caracterizar risco (HADAVAND et al., 2015; MELO et al., 2018).

A investigação também buscou identificar o conhecimento das gestantes em relação a preparação e realização do exame EGB. Assim, identificou-se uma prevalência de 78,6\% para conhecimento satisfatório, achados que se assemelham aos encontrados na literatura, onde observa-se uma prevalência no período puerpério que varia de $50 \%$ a $75 \%$ para conhecimento satisfatório de EGB (JAWOROWSKI et al., 2016; YOUDEN et al., 2005). Acredita-se que esses resultados podem estar relacionados com a homogeneidade das entrevistadas, sendo que a maioria tinha renda superior a 1 salário mínimo e todas tiveram acompanhamento de pré-natal pelo SUS.

Resultados significativos, mostrando associação entre maior conhecimento sobre o exame foram observados nas gestantes com níveis mais altos de escolaridade e maior quantidades de pessoas na casa. Observa-se achados semelhantes com relação a escolaridade, onde segundo estudo realizado no Canadá com 484 mulheres em puerpério, repara-se que as mulheres com elevado nível de escolaridade apresentam maior prevalência de conhecimento satisfatório (YOUDEN et al., 2005). Ainda assim, em um estudo realizado na Polônia, observou-se que as gestantes com maior escolaridade apresentaram maior prevalência de conhecimento satisfatório, embora não tenha relatado significância estatística (JAWOROWSKI et al., 2016). Além disso, encontra-se estudos que associaram o maior nível de escolaridade com a percepção de ameaça por infecção, onde as gestantes com maior conhecimento conseguiram identificar com maior facilidade uma ameaça por infecção (YOUDEN et al., 2005). Sugere-se que quanto maior o nível de escolaridade, maior será o conhecimento satisfatório e a facilidade para interpretar riscos sobre 
EGB. Diante disto, observou-se a importância das informações durante o período pré-natal, estas que elucidam a gestante quanto aos testes realizados durante toda a gestação.

Em relação ao número de pessoas na casa percebe-se que as gestantes que residem com 4 pessoas ou mais demonstraram $20 \%$ mais conhecimento satisfatório. No entanto, não foram encontrados artigos com associações positivas entre o número de pessoas na casa ao conhecimento sobre EGB. Ao mesmo tempo, encontrou-se em estudo uma alta prevalência de conhecimento satisfatório (75\%), onde grande parte das mulheres residiam com três a cinco pessoas $(52,9 \%)$. Por outro lado, estudos mostram que há associação significativa em relação ao estado civil da gestante, embora esta variável não seja significativa neste estudo (JAWOROWSKI et al., 2016). Por isso, acredita-se que o convívio e o apoio de pessoas e companheiros durante a gestação favoreçam o aprendizado da gestante, influenciando no interesse e cuidado durante gestação, estando associados a presença de conhecimento satisfatório.

Referente a renda, percebe-se que as gestantes com mais de um salário mínimo apresentaram $16 \%$ mais conhecimento satisfatório quando comparadas as gestantes com menor renda, embora não tenha apresentado significância estatística. De acordo com Jaworowski et al. (2016), as mulheres com maior nível socioeconômico apresentaram maior conhecimento sobre EGB. Porém, estas também não apresentaram significância $(p=0,063)$. Ao mesmo tempo, percebese que ambos estudos apresentaram associações significativas para escolaridade. Sugere-se que a maior renda facilite o acesso a informações, bem como a escolaridade e ao maior conhecimento.

Segundo Youden et al. (2005), que mediu o conhecimento das mulheres em puerpério sobre infecções que podem ocorrer durante a gestação, observou-se que o conhecimento para EGB foi relativamente menor do que para as outras infecções investigadas. Ainda, notou-se incerteza referente ao exame e risco para EGB, onde várias perguntas foram respondidas com "não tenho certeza". Neste mesmo estudo, percebe-se que em relação a ameaças na saúde do bebê, "muito alto" foi relativamente menos utilizado para infecção com EGB comparado as outras infecções investigadas. Desta forma, apesar da elevada prevalência de conhecimento satisfatório encontrada no presente estudo, sugere-se que não se descarte o fortalecimento em orientações referentes ao exame de EGB no pré-natal.

O pré-natal é um momento ideal para que sejam implementadas medidas de prevenção aos agravos à saúde da mulher e de seu filho. Neste sentido, é de grande relevância a detecção precoce de doenças, preconizadas na realização lúcida de exames como o de EGB (GONDIM et al., 2010). Evidencia-se ainda que estratégias públicas de prevenção do recém-nascido frente ao patógeno são limitadas, uma vez que a recomendação do rastreio universal em todas as gestantes entre a $35^{\mathrm{a}} \mathrm{e}$ $37^{\mathrm{a}}$ semanas não consta como sendo um teste obrigatório pelo Ministério da Saúde, durante o acompanhamento pré-natal (BRASIL, 2012). Considerando que este exame não possui protocolos obrigatórios na clínica pré-natal, tornando-se facultativo o seu requerimento, deve-se considerar a possibilidade de desfecho e impacto negativos na assistência materno-infantil. Assim, torna-se imprescindível o fornecimento de orientações para gestantes quanto à importância, preparação e consequências da realização correta dos exames laboratoriais, principalmente o EGB.

Cabe ressaltar como aspecto limitante na produção deste estudo, a população de gestantes homogênea, possível fator que não possibilitou a comparação com grupos distintos, ou seja, gestantes atendidas no sistema privado. Contempla-se ainda o delineamento transversal, mensurando informações apenas um momento específico, não existindo, portanto, período de seguimento para fortalecer os resultados vigentes sobre o desfecho.

Positivamente, destaca-se que o trabalho, além de descrever a prevalência de Streptococcus agalactiae nas gestantes investigadas, avaliou o conhecimento sobre EGB. Acredita-se que o estudo poderá auxiliar na construção de conhecimento sobre o tema abordado bem como sua importância no meio social, tendo em vista a baixa quantidade de investigações com ênfase ao exposto. Por fim, sugere-se o desenvolvimento de novos estudos que abordem o tema, utilizando delineamento longitudinal. 


\section{Conclusões}

A prevalência de exames positivos para EGB em gestantes de Caxias do Sul-RS encontrouse próxima aos valores de literatura nacional e internacional. Ao mesmo tempo, não foi possível identificar fatores de risco para a presença de EGB. Desse modo, precisam ser desenvolvidos estudos epidemiológicos longitudinais e experimentais que possibilitem a identificação dos determinantes que torna as gestantes mais vulnerárias a contaminação por estes micro-organismos.

De acordo com os resultados aqui apresentados, evidencia-se que o conhecimento sobre EGB tem sido pouco abordado em pesquisas com gestantes. Positivamente, identificou-se uma elevada prevalência de conhecimento satisfatório, a qual mostrou-se associada ao elevado nível de escolaridade e ao maior número de pessoas na casa. Por fim, reforça-se a necessidade de orientações e estratégias públicas que visem à aquisição de conhecimento para a gestante sobre EGB, buscando esclarecer a importância sobre o assunto, melhorar a qualidade dos atendimentos, realização dos exames, saúde das mães e bebês.

\section{Referências}

AREAL, A. et al. A infecção peri-natal por Streptococcus agalactiae pode ser evitada: prevalência da colonização em parturientes no Hospital de S. Marcos, factores de risco e sua relação com a infecção peri-natal. Acta Ped Port.; v. 41, n. 1, p. 16-21, 2010.

ASSEFA, S.; DESTA, K.; LEMA, T. Group B streptococci vaginal colonization and drug susceptibility pattern among pregnant women attending in selected public antenatal care centers in Addis Ababa, Ethiopia. BMC pregnancy and childbirth, v. 18, n. 1, p. 135, 2018.

BARCAITE, E. et al. Prevalence of maternal group B streptococcal colonisation in European countries. Acta obstetricia et gynecologica Scandinavica, v. 87, n. 3, p. 260-271, 2008.

BRASIL. MINISTÉRIO DA SAÚDE. Assistência pré-natal: normas e manuais técnicos: Secretaria de Políticas de Saúde, Ministério da Saúde Brasília 2000.

BRASIL. MINISTÉRIO DA SAÚDE, S. DE ATENÇÃO À SAÚDE, DEPARTAMENTO DE AÇÕES PROGRAMÁTICAS E ESTRATÉGICAS. Atenção à saúde do recém-nascido: guia para os profissionais de saúde: Ed. Ministério da Saúde Brasília 2011.

BRASIL. MINISTÉRIO DA SAÚDE. SECRETARIA DE ATENÇÃO A SAÚDE. DEPARTAMENTO DE CIÊNCIA E TECNOLOGIA DEPARTAMENTO DE AÇÕES PROGRAMÁTICAS. Gestação de Alto Risco: manual técnico: MS Brasília (DF) 2012.

CONTROL, C. F. D.; PREVENTION. Sexually transmitted diseases treatment guidelines, 2010. Annals of Emergency Medicine, v. 58, n. 1, p. 67-68, 2011.

DARABI, R. et al. The prevalence and risk factors of group B streptococcus colonization in Iranian pregnant women. Electronic physician, v. 9, n. 5, p. 4399, 2017.

ELBARADIE, S. M.; MAHMOUD, M.; FARID, M. Maternal and neonatal screening for Group B streptococci by SCP B gene based PCR: a preliminary study. Indian journal of medical microbiology, v. 27, n. 1, p. 17, 2009. 
FUNÇÃO, J. M.; NARCHI, N. Z. Pesquisa do estreptococo do Grupo B em gestantes da Zona Leste de São Paulo. Revista da Escola de Enfermagem da USP, v. 47, n. 1, p. 22-29, 2013.

GONDIM, A. N. C. et al. Fast HIV screening test for parturients: a quantitative study. Online Brazilian Journal of Nursing, v. 9, n. 2, 2010.

HADAVAND, S. et al. Frequency of Group B Streptococcal colonization in pregnant women aged 35-37 weeks in clinical centers of Shahed University, Tehran, Iran. Iranian journal of pathology, v. 10, n. 2, p. 120, 2015.

HALL, J. et al. Maternal disease with group B Streptococcus and serotype distribution worldwide: systematic review and meta-analyses. Clinical infectious diseases, v. 65, n. suppl_2, p. S112S124, 2017.

HUNG, L.-C. et al. Risk factors for neonatal early-onset group B streptococcus-related diseases after the implementation of a universal screening program in Taiwan. BMC public health, v. 18, n. 1, p. 438, 2018.

JAWOROWSKI, A. et al. The level of implementation of the prevention program of group B Streptococcal infection during pregnancy and in newborns in accordance to the recommendations of the Polish Gynaecological Society-questionnaire survey. Przeglad lekarski, v. 73, n. 9, p. 632636, 2016.

JI, W. et al. Colonization prevalence and antibiotic susceptibility of Group B Streptococcus in pregnant women over a 6-year period in Dongguan, China. PloS one, v. 12, n. 8, p. e0183083, 2017.

KRUK, C. R. et al. Epidemiologic profile of Streptococcus agalactiae colonization in pregnant women attending prenatal care in a city of southern of Brazil. Brazilian Journal of Infectious Diseases, v. 17, n. 6, p. 722-723, 2013.

LINHARES, J. J. et al. Prevalence of the colonization by Streptococcus agalactiae in pregnant women from a maternity in Ceará, Brazil, correlating with perinatal outcomes. Revista Brasileira de Ginecologia e Obstetrícia, v. 33, n. 12, p. 395-400, 2011.

MELO, S. C. C. S. D. et al. Prevalence of Streptococcus agalactiae colonization in pregnant women from the 18th Health Region of Paraná State. Revista do Instituto de Medicina Tropical de São Paulo, v. 60, 2018.

MENGIST, H. M. et al. Prevalence and drug susceptibility pattern of group B Streptococci (GBS) among pregnant women attending antenatal care (ANC) in Nekemte Referral Hospital (NRH), Nekemte, Ethiopia. BMC research notes, v. 10, n. 1, p. 388, 2017.

MORALEDA, C. et al. Prevalence, antimicrobial resistance and serotype distribution of group B streptococcus isolated among pregnant women and newborns in Rabat, Morocco. Journal of medical microbiology, 2018.

PAVANATTO, A.; ALVES, L. M. S. Programa de humanização no pré natal e nascimento: indicadores e práticas das enfermeiras. Revista de Enfermagem da UFSM, v. 4, n. 4, p. 761-770, 2014. 
PENELAS, N.; PINHEIRO, S. Prevalência da Colonização de Strep Grupo B numa população de Grávidas do distrito de Vila Real. Acta Farmacêutica Portuguesa, v. 5, n. 1, p. 75-79, 2016.

PINTO, A. M. et al. Incidence and serotype characterisation of Streptococcus agalactiae in a Portuguese hospital. Journal of Clinical Pathology, p. jclinpath-2017-204646, 2017.

RAUSCH, A.-V. et al. Group B Streptococcus colonization in pregnancy: prevalence and prevention strategies of neonatal sepsis. Journal of perinatal medicine, v. 37, n. 2, p. 124-129, 2009.

RICK, A.-M. et al. Group B streptococci colonization in pregnant guatemalan women: prevalence, risk factors, and vaginal microbiome. In: Open forum infectious diseases. Oxford University Press, 2017.

SANTHANAM, S. et al. Prevalence of group B Streptococcal colonization among pregnant women and neonates in a tertiary hospital in India. Journal of the Turkish German Gynecological Association, v. 18, n. 4, p. 181-184, 2017.

SHARMILA, V. et al. Genital tract group B streptococcal colonization in pregnant women: a South Indian perspective. The Journal of Infection in Developing Countries, v. 5, n. 08, p. 592595, 2011.

SIGAÚQUE, B. et al. Invasive bacterial disease trends and characterization of group B streptococcal isolates among young infants in southern Mozambique, 2001-2015. PloS one, v. 13, n. 1, p. e0191193-e0191193, 2018.

VACILOTO, E. et al. A survey of the incidence of neonatal sepsis by group B Streptococcus during a decade in a Brazilian maternity hospital. Brazilian Journal of Infectious Diseases, v. 6, n. 2, p. 55-62, 2002.

VORNHAGEN, J.; WALDORF, K. M. A.; RAJAGOPAL, L. Perinatal group B streptococcal infections: virulence factors, immunity, and prevention strategies. Trends in microbiology, v. 25, n. 11, p. 919-931, 2017.

WOLLHEIM, C. et al. Group B Streptococcus detection in pregnant women via culture and PCR methods. Revista da Sociedade Brasileira de Medicina Tropical, v. 50, n. 2, p. 179-183, 2017.

YOUDEN, L. et al. Group B streptococcal testing during pregnancy: survey of postpartum women and audit of current prenatal screening practices. Journal of Obstetrics and Gynaecology Canada, v. 27, n. 11, p. 1006-1012, 2005. 